\title{
Stability and Stabilization of Delayed Neural Networks with Hybrid Impulses
}

\author{
Kefa Zou $\mathbb{D}^{1},{ }^{1}$ Xuechen Li $\left(\mathbb{D},{ }^{1}\right.$ Nan Wang $\mathbb{D}^{1},{ }^{1}$ Jungang Lou $\mathbb{D},{ }^{2}$ and Jianquan Lu $\mathbb{D}^{3,4}$ \\ ${ }^{1}$ School of Science, Xuchang University, Xuchang 461000, China \\ ${ }^{2}$ Zhejiang Province Key Laboratory of Smart Management \& Application of Modern Agricultural Resources, \\ School of Information Engineering, Huzhou University, Huzhou 313000, China \\ ${ }^{3}$ School of Mathematics, Southeast University, Nanjing 210096, China \\ ${ }^{4}$ Key Laboratory of Complex Systems and Intelligent Computing in Universities of Shandong, \\ School of Automation and Electrical Engineering, Linyi University, Linyi 276005, China
}

Correspondence should be addressed to Jianquan Lu; jqluma@seu.edu.cn

Received 16 July 2020; Revised 11 October 2020; Accepted 4 November 2020; Published 21 November 2020

Academic Editor: Giacomo Innocenti

Copyright (c) 2020 Kefa Zou et al. This is an open access article distributed under the Creative Commons Attribution License, which permits unrestricted use, distribution, and reproduction in any medium, provided the original work is properly cited.

In this paper, the stability and stabilization issues for a class of delayed neural networks with time-varying hybrid impulses are investigated. The hybrid effect of two types of impulses including both stabilizing and destabilizing impulses is considered simultaneously in the analysis of systems. To characterize the occurrence features of impulses, the concepts of average impulse interval and average impulse strength are employed. Based on the analysis of stability, a pinning impulsive controller which can ensure the global exponential stability of the studied neural networks is designed by pinning a small fraction of neurons. Finally, two numerical examples are given to illustrate the effectiveness of the proposed control schemes for delayed neural networks with hybrid impulses.

\section{Introduction}

During the past few decades, dynamic networks have been systematically studied due to their broad application background in different areas [1-9]. In recent years, in order to cater to the specific needs of modeling various practical systems, many kinds of dynamic networks with special structures have been developed, for example, [10-13] and the references therein. When networks encounter transient disturbance or abrupt dynamic variation in various instants, systems may display switching or impulsive behaviors $[14,15]$. Consequently, impulsive neural networks, which can model various electronic or biological networks encountering instantaneous and abrupt changes frequently, have been extensively investigated in various fields of science and engineering [16-19]. On the contrary, time delays frequently appear in various dynamical systems $[20,21]$. The existence of time delays in neural networks may induce more complex dynamical behaviors such as instability, oscillations, and chaos [22-27]. Therefore, it is necessary to investigate effects of time delays and impulses on the stability of neural networks.

Generally, impulses can be divided into two categories according to their impact on systems. It is supposed that the impulses are destabilizing if the impulses can potentially destroy the stability of dynamical systems, while the impulses are considered to be stabilizing if they are potentially beneficial for the stabilization of dynamical systems. Stabilizing impulses can be considered as impulsive controllers, which can enhance the stabilization of dynamical systems. In the last several decades, stabilizing impulses and destabilizing impulses have been studied by a great many scholars [28-31]. At the same time, the impulsive control method has received many researchers' attention (see [32-36] and references therein). Particularly, stability or stabilization issue for dynamical systems with delays and impulses was investigated in [37-41] and references therein. In [42], a unified synchronization criterion for impulsive dynamical 
networks subject to desynchronizing or synchronizing impulses was derived by using the average impulsive interval approach. In [43], some adequate conditions that can ensure the exponential synchronization of inertial memristor-based neural networks with time delay were given by utilizing the average impulsive interval approach. In [44], the pinning impulsive control strategy was proposed. By utilizing the Lyapunov method combined with the comparison principle, pinning stabilization of probabilistic Boolean networks subject to time delays was investigated in [45]. Synchronization problem for stochastic neural networks was studied by impulsively controlling partial states in [46]. Recently, networks with hybrid impulses were explored in $[47,48]$. To reduce conservativeness, a novel piecewise Lyapunov-Krasovskii functional (LKF) was constructed by introducing a line-integral type Lyapunov function and some useful terms that take full advantage of the available information about the actual sampling pattern in [49].

In this paper, the concepts of average impulsive interval and average impulsive strength are introduced to characterize the features of hybrid impulses. Then, based on the Lyapunov method combined with the utilization of proper mathematical analysis techniques, the stability analysis for neural networks with time-varying delays and hybrid impulses is carried out. Under this circumstance, the classification of stabilizing and destabilizing impulses is not taken into account; just the overall effect of the impulses is taken into consideration. Furthermore, a pinning impulsive controller design procedure for the stabilization of the investigated neural networks is proposed based on the above analysis. In this controller, only a small fraction of variables is impulsively controlled to ensure the global and exponential stability of neural networks. The main contributions of this paper are summarized as follows: (1) a new concept of "average impulsive strength," which can be used to characterize much wider range of impulsive sequences, is introduced to describe the hybrid impulses investigated in this paper. (2) By virtue of some proper disposing techniques relevant to average impulsive interval and average impulsive strength in the proof process of the main results, less conservative results can thereafter be obtained. (3) In consideration of the advantages of low cost and high efficiency of the pinning controller, the strategy that selects only a small fraction of neurons for impulsive control is adopted to achieve the stabilization of the delayed neural networks.

The remainder of this paper is arranged as follows: in Section 2, we propose the problem of stability and stabilization of delayed neural networks with hybrid impulses and give some necessary preliminaries. In Section 3, a criterion for determining the stability of delayed neural networks with hybrid impulses is established, and then a pinning impulsive controller is designed to stabilize delayed neural networks. In Section 4, numerical examples are given to illustrate our theoretical results. Finally, Section 5 presents the conclusion.

Notation 1 . The standard notations are used in this paper. $\mathbb{R}^{+}$and $\mathbb{R}^{n}$ denote the set of nonnegative real numbers and the $n$-dimensional Euclidean space. $\mathbb{N}_{+}$denotes the set of positive integers. The superscript " $T$ " represents the transpose of the matrix or vector. For $x \in \mathbb{R}^{n},|x|$ denotes the Euclidean norm of $x$. For matrix $A \in \mathbb{R}^{n},\|A\|=\sqrt{\lambda_{\max }\left(A^{T} A\right)}$, where $\lambda_{\max }(\cdot)$ represents the largest eigenvalue. $\operatorname{diag}(\cdots)$ stands for a block-diagonal matrix. Given $\tau>0, C\left([-\tau, 0], \mathbb{R}^{n}\right)$ denotes the family of continuous functions from $[-\tau, 0]$ to $\mathbb{R}^{n}$.

\section{Preliminaries}

In this section, some preliminaries including model formulation, lemmas, and definitions are presented.

Consider the following neural network:

$$
\dot{x}(t)=C x(t)+B g(x(t))+D g(x(t-\tau(t)))+I,
$$

where $x(t)=\left(x_{1}(t), x_{2}(t), \ldots, x_{n}(t)\right)^{T} \in \mathbb{R}^{n}$ is the state vector associated with the neurons; $C=\operatorname{diag}\left(c_{1}, c_{2}\right.$, $\left.\ldots, c_{n}\right)<0$ is the self-feedback matrix; $B=\left(b_{i j}\right)_{n \times n}$ and $D=$ $\left(d_{i j}\right)_{n \times n}$ are the connection weight matrices; $g(x(t))=\left(g_{1}(x(t)), g_{2}(x(t)), \ldots, g_{n}(x(t))\right)^{T}$ denotes the activation function of the neurons; $I \in \mathbb{R}^{n}$ signifies constant external input; and $\tau(t)$ represents the time-varying delays that satisfies $0 \leq \tau(t) \leq \tau$. For the nonlinear function $g(\cdot)$, we have the following assumption.

Assumption 1. Assume that $g_{i}(\cdot)(i=1,2, \ldots, n)$ are globally Lipschitz continuous functions, i.e., there exist constants $l_{i}>0(i=1,2, \ldots, n)$ such that

$$
\left|g_{i}\left(x_{1}\right)-g_{i}\left(x_{2}\right)\right| \leq l_{i}\left|x_{1}-x_{2}\right|, \quad \text { for any } x_{1}, x_{2} \in \mathbb{R} .
$$

Denote $L=\operatorname{diag}\left(l_{1}, l_{2}, \ldots, l_{n}\right)$.

Let $x^{*}$ be the equilibrium point of (1). For convenience, we can shift the intended equilibrium $x^{*}$ to be original by letting $y=x-x^{*}$, and then system (1) can be transformed into

$$
\dot{y}(t)=C y(t)+B f(y(t))+D f(y(t-\tau(t))),
$$

where $y(t)=\left(y_{1}(t), y_{2}(t), \ldots, y_{n}(t)\right)^{T} \in \mathbb{R}^{n}$ is the state vector of the transformed system. It follows from (2) that the function $f(y)=g\left(y+x^{*}\right)-g\left(x^{*}\right)$ satisfies

$$
\left|f_{i}\left(x_{1}\right)-f_{i}\left(x_{2}\right)\right| \leq l_{i}\left|x_{1}-x_{2}\right|, \quad \text { for any } x_{1}, x_{2} \in \mathbb{R} .
$$

In consideration of the time-varying impulse effects, the impulsive delayed neural network can be obtained in the following form:

$$
\begin{cases}\dot{y}(t)=C y(t)+B f(y(t))+D f(y(t-\tau(t))), & t \neq t_{k}, \\ y\left(t_{k}^{+}\right)=\alpha_{k} y\left(t_{k}^{-}\right), & k \in \mathbb{N}_{+},\end{cases}
$$

where $\left\{t_{1}, t_{2}, t_{3}, \ldots,\right\}$ is a sequence of strictly increasing impulsive moments. $\alpha_{k} \in \mathbb{R}$ represents the strength of impulses. We assume that $y(t)$ is right-continuous at $t=t_{k}$, i.e., $y\left(t_{k}\right)=y\left(t_{k}^{+}\right)$. Hence, the solutions of (5) are piecewise right-hand continuous functions which are discontinuous at $t=t_{k}$ for $k \in \mathbb{N}_{+}$. The initial condition of (5) is given by $y(t)=\phi(t) \in C\left([-\tau, 0], \mathbb{R}^{n}\right)$. 
Definition 1. (average impulsive interval; see [42]). $T_{a}$ is called the average impulsive interval of the impulsive sequence $\zeta=\left\{t_{1}, t_{2}, \ldots,\right\}$ if

$$
\frac{T-t}{T_{a}}-N_{0} \leq N_{\zeta}(T, t) \leq \frac{T-t}{T_{a}}+N_{0}, \quad \text { for all } T \geq t \geq 0,
$$

where $N_{0}$ is a positive integer and $T_{a}$ is a positive number. $N_{\zeta}(T, t)$ denotes the impulsive times of the impulsive sequence $\zeta$ in the time interval $(t, T)$.

Definition 2 (average impulsive strength). $\alpha$ is called the average impulsive strength of the sequence

$$
\begin{gathered}
\zeta(T, t)=\left\{t_{l}, t_{l+1}, \ldots, t_{l+N_{\zeta}(T, t)-1}\right\} \text { for all } T \geq t \geq 0 \text { if } \\
\frac{\left|\alpha_{N_{\zeta}(t, 0)}\right|+\left|\alpha_{N_{\zeta}(t, 0)+1}\right|+\cdots+\left|\alpha_{N_{\zeta}(T, 0)-1}\right|}{\alpha}-N_{1} \leq N_{\zeta}(T, t) \\
\leq \frac{\left|\alpha_{N_{\zeta}(t, 0)}\right|+\left|\alpha_{N_{\zeta}(t, 0)+1}\right|+\cdots+\left|\alpha_{N_{\zeta}(T, 0)-1}\right|}{\alpha}+N_{1},
\end{gathered}
$$

where $N_{1}$ is a positive integer and $\alpha$ is a positive number. $N_{\zeta}(T, t)$ denotes the impulsive times of the impulsive sequence $\zeta$ in the time interval $(t, T)$.

Furthermore, inequality (7) implies that

$$
\begin{aligned}
\alpha\left(1-\frac{N_{1}}{N_{\zeta}(T, t)}\right) & \leq \frac{\left|\alpha_{N_{\zeta}(t, 0)}\right|+\left|\alpha_{N_{\zeta}(t, 0)+1}\right|+\cdots+\left|\alpha_{N_{\zeta}(T, 0)-1}\right|}{N_{\zeta}(T, t)} \\
& \leq \alpha\left(1+\frac{N_{1}}{N_{\zeta}(T, t)}\right) .
\end{aligned}
$$

Remark 1. The concept of average impulsive gain was proposed in [48], where the problem of the globally exponential synchronization of coupled neural networks with hybrid impulses was investigated. However, when the timevarying delays are taken into consideration simultaneously, this idea may fail to be applied to the analysis of the stability of delayed neural networks with hybrid impulses. To deal with the difficulties coming from time-varying delays, a new concept named "average impulsive strength" is introduced in this paper. The conditions of our concept are more strict, so it can ensure the establishment of the inequality in the comparison principle.

Definition 3. Impulsive neural networks (5) are said to be globally exponentially stable if there exist constants $M>0, \lambda>0$, and $T_{0}>0$ such that, for any initial values, $|y(t)|^{2} \leq \mathrm{Me}^{-\lambda t}$ holds for all $t \geq T_{0}$.

Lemma 1 (see [42]). For any vectors $x, y \in \mathbb{R}^{n}$, scale $\epsilon>0$, and positive definite matrix $Q \in \mathbb{R}^{n \times n}$, the following inequality holds: $2 x^{T} y \leq \varepsilon x^{T} Q x+\varepsilon^{-1} y^{T} Q^{-1} y$.
Lemma 2 (see [35]). m+1 Let $0 \leq \tau_{i}(t) \leq \tau$. $F\left(t, u, \bar{u}_{1}, \bar{u}_{2}, \ldots, \bar{u}_{m}\right): \mathbb{R}^{+} \times \overbrace{\mathbb{R} \times \cdots \times \mathbb{R}}^{\longrightarrow} \mathbb{R}$ is nondecreasing in $\bar{u}_{i}$ for each fixed $\left(t, u, \bar{u}_{1}, \ldots\right.$, $\left.\bar{u}_{i-1}, \bar{u}_{i-2}, \ldots, \bar{u}_{m}\right), i=1,2, \ldots, m$, and $I_{k}(u): \mathbb{R} \longrightarrow \mathbb{R}$ is nondecreasing in $u$.

Suppose that

$$
\begin{aligned}
& \left\{\begin{array}{l}
D^{+} u(t) \leq F\left(t, u(t), u\left(t-\tau_{1}(t)\right), \ldots, u\left(t-\tau_{m}(t)\right)\right), \\
u\left(t_{k}^{+}\right) \leq I_{k}\left(u\left(t_{k}^{-}\right)\right), \quad k \in \mathbb{N}_{+},
\end{array}\right. \\
& \left\{\begin{array}{l}
D^{+} v(t)>F\left(t, v(t), v\left(t-\tau_{1}(t)\right), \ldots, v\left(t-\tau_{m}(t)\right)\right), \\
v\left(t_{k}^{+}\right) \geq I_{k}\left(v\left(t_{k}^{-}\right)\right), \quad k \in \mathbb{N}_{+} .
\end{array}\right.
\end{aligned}
$$

Then, $u(t) \leq v(t)$, for $-\tau \leq t \leq 0$, implies that $u(t) \leq v(t)$, for $t \geq 0$.

\section{Main Results}

In this section, we will analyze the global exponential stability of delayed neural networks with hybrid impulses including both destabilizing and stabilizing impulses.

Theorem 1. Consider time-varying neural network (5) with hybrid impulses including both destabilizing and stabilizing impulses. Suppose that Assumption 1 holds and that the average impulsive interval of the impulsive sequence $\zeta=\left\{t_{1}, t_{2}, \ldots,\right\}$ is $T_{a}$. Then, neural networks (5) with hybrid impulses are globally exponentially stable if the following inequality holds:

$$
\eta_{1}+M_{0} q<0
$$

where $\quad \eta_{1}=p+2 \ln \alpha / T_{a}, p=\lambda_{\max }\left(C+C^{T}+B B^{T}+\right.$ $\left.L^{T} L+D D^{T}\right), q=\lambda_{\max }\left(L^{T} L\right)$, and $M_{0}$ is a constant satisfying the following condition: when $\alpha \geq 1, M_{0}=e^{2 N_{1}} \alpha^{2 N_{0}}$; otherwise, $M_{0}=e^{2 N_{1}} \alpha^{-2 N_{0}}$.

Proof. Consider the Lyapunov function $V(t)=y^{T}(t) y(t)$. Then, the derivative of $V(t)$ along the trajectories of system (5) can be obtained as follows:

$$
\begin{aligned}
D^{+} V(t)= & y^{T}(t)\left(C+C^{T}\right) y(t)+2 y^{T}(t) B f(y(t)) \\
& +2 y^{T}(t) D f(y(t-\tau(t))), \quad t \in\left(t_{k-1}, t_{k}\right], k \in \mathbb{N}_{+} .
\end{aligned}
$$

By Lemma 1, one obtains

$$
\begin{aligned}
2 y^{T}(t) B f(y(t)) & \leq y^{T}(t) B B^{T} y(t)+f^{T}(y(t)) f(y(t)) \\
& \leq y^{T}(t) B B^{T} y(t)+y^{T}(t) L^{T} L y(t),
\end{aligned}
$$

$$
\begin{aligned}
\left.2 y^{T}(t) D f(t-\tau(t))\right) \leq & y^{T}(t) D D^{T} y(t)+f^{T}(y(t-\tau(t))) \\
& \cdot f(y(t-\tau(t))) \leq y^{T}(t) D D^{T} y(t) \\
& +y^{T}(t-\tau(t)) L^{T} L y(t-\tau(t)) .
\end{aligned}
$$

From (11) to (13), it follows that 


$$
\begin{aligned}
D^{+} V(t) \leq & y^{T}(t)\left(C+C^{T}+B B^{T}+L^{T} L+D D^{T}\right) y(t) \\
& +y^{T}(t-\tau(t)) L^{T} L y(t-\tau(t)) \\
\leq & \lambda_{\max }\left(C+C^{T}+B B^{T}+L^{T} L+D D^{T}\right) V(t) \\
+ & \lambda_{\max }\left(L^{T} L\right) V(t-\tau(t))=p V(t)+q V(t-\tau(t)), \\
& t \in\left(t_{k-1}, t_{k}\right], k \in \mathbb{N}_{+} .
\end{aligned}
$$

For $t=t_{k}$, one has

$$
V\left(t_{k}^{+}\right)=y^{T}\left(t_{k}^{+}\right) y\left(t_{k}^{+}\right)=\alpha_{k}^{2} y^{T}\left(t_{k}^{-}\right) y\left(t_{k}^{-}\right)=\alpha_{k}^{2} V\left(t_{k}^{-}\right) .
$$

For any $\varepsilon>0$, let $\nu(t)$ be a unique solution of the following impulsive delay system:

$$
\begin{cases}\dot{v}(t)=p \nu(t)+q \nu(t-\tau(t))+\varepsilon, & t \neq t_{k}, \\ \left.\nu\left(t_{k}^{-}\right)=\alpha_{k}^{2} \nu\left(t_{k}^{-}\right)\right), & t=t_{k} k \in \mathbb{N}_{+}, \\ \nu(t)=|\phi(t)|^{2}, & -\tau \leq t \leq 0 .\end{cases}
$$

According to Lemma 2, one gets

$$
\nu(t) \geq V(t), \quad t \geq 0 .
$$

By the formula for the variation of parameters, it follows from (16) that

$$
\nu(t)=w(t, 0) \nu(0)+\int_{0}^{t} w(t, s)[q v(s-\tau(s)+\varepsilon] \mathrm{d} s,
$$

where $w(t, s), t>s \geq 0$, is the Cauchy matrix of the linear system

$$
\begin{cases}\dot{z}(t)=p z(t), & t \neq t_{k}, \\ \left.z\left(t_{k}^{+}\right)=\alpha_{k}^{2} z\left(t_{k}^{-}\right)\right), & t=t_{k} k \in \mathbb{N}_{+} .\end{cases}
$$

According to the representation of the Cauchy matrix, we can obtain the following estimation: if $N_{\zeta}(t, s)>0$, one has

$$
\begin{aligned}
w(t, s) & =e^{p(t-s)} \prod_{s<t_{k} \leq t} \alpha_{k}^{2} \\
& \leq e^{p(t-s)}\left(\frac{\left|\alpha_{N_{\zeta}(s, 0)}\right|+\left|\alpha_{N_{\zeta}(s, 0)+1}\right|+\cdots+\left|\alpha_{N_{\zeta}(t-s)-1}\right|}{N_{\zeta}(t-s)}\right)^{2 N_{\zeta}(t-s)} \\
& \leq e^{p(t-s)}\left[\alpha\left(1+\frac{N_{1}}{N_{\zeta}(t-s)}\right)\right]^{2 N_{\zeta}(t-s)} \\
& \leq e^{2 N_{1}} e^{p(t-s)} \alpha^{2 N_{\zeta}(t-s)} .
\end{aligned}
$$

Otherwise, when $N_{\zeta}(t, s)>0$, one also has

$$
w(t, s)=e^{p(t-s)} \leq e^{2 N_{1}} e^{p(t-s)} \alpha^{2 N_{\zeta}(t, s)} .
$$

Since the average impulsive interval of the impulsive sequence $\zeta=\left\{t_{1}, t_{2}, \ldots,\right\}$ is equal to $T_{a}$, we have
$\frac{t-s}{T_{a}}-N_{0} \leq N_{\zeta}(t, s) \leq \frac{t-s}{T_{a}}+N_{0}, \quad$ for all $t \geq s \geq 0$.

If $\alpha \geq 1$, it follows from (20) to (22) that

$w(t, s) \leq e^{2 N_{1}} e^{p(t-s)} \alpha^{2\left(t-s / T_{a}+N_{0}\right)} \leq \alpha^{2 N_{0}} e^{2 N_{1}} e^{\left(p+2 \ln \alpha / T_{a}\right)(t-s)}$.

Similarly, when $\alpha<1$, it follows from (20) and (22) that $w(t, s) \leq e^{2 N_{1}} e^{p(t-s)} \alpha^{2\left(t-s / T_{a}-N_{0}\right)} \leq \alpha^{-2 N_{0}} e^{2 N_{1}} e^{\left(p+2 \ln \alpha / T_{a}\right)(t-s)}$.

Letting $\varepsilon \longrightarrow 0$ and summarizing inequalities (23) and (24) give that there exists constant $M_{0}=\max \left\{\alpha^{2 N_{0}} e^{2 N_{1}}, \alpha^{-2 N_{0}} e^{2 N_{1}}\right\}$ such that

$$
w(t, s)<M_{0} e^{\eta_{1}(t-s)},
$$

where $\eta_{1}=p+2 \ln \alpha / T_{a}$.

Let $\eta=M_{0} \sup _{-\tau \leq s \leq 0}|\phi(s)|^{2}$. From (18) and (25), one obtains

$$
\nu(t) \leq \eta e^{\eta_{1} t}+\int_{0}^{t} M_{0} e^{\eta_{1}(t-s)}[q \nu(s-\tau(s))+\varepsilon] \mathrm{d} s .
$$

Define $h(v)=v+\eta_{1}+M_{0} q e^{v \tau}$. It follows from (10) that $h(0)<0$. Since $h(+\infty)=+\infty$ and $\dot{h}(v)>0$, there exists a unique $\lambda>0$ such that

$$
\lambda+\eta_{1}+M_{0} q e^{\lambda \tau}=0 .
$$

On the contrary, it is obvious from (10) that $M_{0}^{-1} \eta_{1}+q<0$. Hence,

$$
\nu(t)=|\phi(t)|^{2} \leq \eta<\eta e^{-\lambda t}-\frac{\varepsilon}{M_{0}^{-1} \eta_{1}+q}, \quad-\tau \leq t \leq 0 .
$$

Then, we claim

$$
\nu(t)<\eta e^{-\lambda t}-\frac{\varepsilon}{M_{0}^{-1} \eta_{1}+q} .
$$

If inequality (29) is not true, there exists $t^{*}>0$ such that

$$
\begin{aligned}
& \nu\left(t^{*}\right) \geq \eta e^{-\lambda t^{*}}-\frac{\varepsilon}{M_{0}^{-1} \eta_{1}+q}, \\
& v(t)<\eta e^{-\lambda t}-\frac{\varepsilon}{M_{0}^{-1} \eta_{1}+q}, \quad t<t^{*} .
\end{aligned}
$$

From (26) to (31), we have

$$
\begin{aligned}
\nu\left(t^{*}\right) \leq & \eta e^{\eta_{1} t^{*}}+\int_{0}^{t^{*}} M_{0} e^{\eta_{1}\left(t^{*}-s\right)}[q \nu(s-\tau(s))+\varepsilon] \mathrm{d} s \\
<<e^{\eta_{1} t^{*}} & \left\{\eta-\frac{\varepsilon}{M_{0}^{-1} \eta_{1}+q}+\int_{0}^{t^{*}} M_{0} e^{-\eta_{1} s}\right. \\
\cdot & {\left.\left[q\left(\eta e^{-\lambda(s-\tau(s))}-\frac{\varepsilon}{M_{0}^{-1} \eta_{1}+q}\right)+\varepsilon\right] \mathrm{d} s\right\} . }
\end{aligned}
$$

It is derived from (27) and (32) that 


$$
\begin{aligned}
v\left(t^{*}\right)< & e^{\eta_{1} t^{*}}\left\{\eta-\frac{\varepsilon}{M_{0}^{-1} \eta_{1}+q}+\eta e^{-\left(\eta_{1}+\lambda\right) t^{*}}\right. \\
& \left.-\eta-\frac{\varepsilon}{M_{0}^{-1} \eta_{1}+q} e^{-\eta_{1} t^{*}}+\frac{\varepsilon}{M_{0}^{-1} \eta_{1}+q}\right\} \\
= & \eta e^{-\lambda t^{*}}-\frac{\varepsilon}{M_{0}^{-1} \eta_{1}+q}
\end{aligned}
$$

which contradicts with (30), and so, (29) holds. Letting $\varepsilon \longrightarrow 0$, one gets from (17) that

$$
V(t) \leq \nu(t) \leq \eta e^{-\lambda t}
$$

By Definition 3, the solution $y(t)$ of impulsive neural networks (5) is exponentially stable. The proof is hence completed.

Remark 2. The concepts of average impulse interval [42, 43] and average impulsive strength are employed to characterize the features of hybrid impulses. The classification of stabilizing and destabilizing impulses is not taken into account; just the overall effect of the impulses is taken into consideration. By utilizing the comparison principle [35, 44], Lyapunov theory $[35,42-44]$, Young's inequality technique $[42,43,48]$, average impulsive interval approach [42, 43], and the concept of average impulsive strength, Theorem 1 presenting conditions of ensuring the global exponential stability of delayed neural networks (5) is derived.

In the following section, a pinning impulsive controller will be designed to globally and exponentially stabilize neural networks (5). In order to drive system (5) into the equilibrium point $x^{*}$, the following impulsive controller is constructed for $l$ partial variables $(l<n)$ :

$$
u_{i}\left(t_{k}\right)= \begin{cases}0, & i \notin \mathscr{D}\left(t_{k}\right), \\ \sum_{k=1}^{+\infty} \mu y_{i}(t) \delta\left(t-t_{k}\right), & i \in \mathscr{D}\left(t_{k}\right),\end{cases}
$$

where $\mu \in(-2,-1) \cup(-1,0)$ is a constant, which means that the impulsive effects can be used to stabilize the delayed neural networks; the index set of $l$ partial variables $\mathscr{D}\left(t_{k}\right)$ which should be impulsively controlled is defined as follows: at time instant $t_{k}$, for the error of states of the partial variables $y_{1}\left(t_{k}\right), y_{2}\left(t_{k}\right), \ldots, y_{n}\left(t_{k}\right)$, one can reorder the states such that $\left|y_{p_{1}}\left(t_{k}\right)\right| \geq\left|y_{p_{2}}\left(t_{k}\right)\right| \geq \cdots \geq\left|y_{p_{n}}\left(t_{k}\right)\right|$.

Then, the index set of $l$ controlled variables $\mathscr{D}\left(t_{k}\right)$ is defined as $\mathscr{D}\left(t_{k}\right)=\left\{p_{1}, p_{2}, \ldots, p_{l}\right\}$. After adding pinning impulsive controller (35) to the variables $\mathscr{D}\left(t_{k}\right)$, the controlled delayed neural networks can be rewritten as follows:

$$
\begin{aligned}
\dot{y}(t) & =C y(t)+B f(y(t))+D f(y(t-\tau(t))), t \neq t_{k}, \\
y_{i}\left(t_{k}^{+}\right) & =\mu y_{i}\left(t_{k}^{-}\right)+y_{i}\left(t_{k}^{-}\right), \quad i \in \mathscr{D}\left(t_{k}\right), \\
y\left(t_{k}^{+}\right) & =y_{i}\left(t_{k}^{-}\right), \quad i \notin \mathscr{D}\left(t_{k}\right) .
\end{aligned}
$$

The initial conditions of delayed neural networks (36) are given by

$$
y_{i}(t)=\phi_{i}(t) \quad-\tau \leq t \leq 0(i=1,2, \ldots, n),
$$

where $\phi_{i}(t) \in C([-\tau, 0], \mathbb{R})$ with $C([-\tau, 0], \mathbb{R})$ being the set of continuous functions from $[-\tau, 0]$ to $\mathbb{R}$.

Theorem 2. Let $\rho=n+l \mu(\mu+2) / n \in(0,1)$. Suppose that Assumption 1 holds and that the average impulsive interval $\zeta=\left\{t_{1}, t_{2}, \ldots,\right\}$ is $T_{a}$. Then, neural networks (36) are globally exponentially stabilized to the state $x^{*}$ by pinning controller (35) if the following inequality holds:

$$
\eta_{2}+M_{1} q<0
$$

where $\eta_{2}=p+\ln \rho / T_{a}, p=\lambda_{\max }\left(C+C^{T}+B B^{T}+L^{T} L+\right.$ $\left.D D^{T}\right), q=\lambda_{\max }\left(L^{T} L\right)$, and $M_{1}=\rho^{-N_{0}}$.

Proof. Construct a Lyapunov function in the form of

$$
V(t)=y^{T}(t) y(t)=\sum_{i=1}^{n} y_{i}^{2}(t) .
$$

By a similar analysis as Theorem 1 , for any $t \in\left[t_{k-1}, t_{k}\right)$, taking the derivative of $V(t)$ along the solution of (36) gives that

$$
D^{+} V(t) \leq p V(t)+q V(t-\tau(t)) .
$$

For any $k \in \mathbb{N}_{+}$, we have

$$
\begin{aligned}
V\left(t_{k}^{+}\right)= & \sum_{i=1}^{n} y_{i}^{T}\left(t_{k}^{+}\right) y_{i}\left(t_{k}^{+}\right)=\sum_{i \in \mathscr{D}\left(t_{k}\right)}(1+\mu)^{2} y_{i}^{T}\left(t_{k}^{-}\right) y_{i}\left(t_{k}^{-}\right) \\
& +\sum_{i \notin \mathscr{D}\left(t_{k}\right)} y_{i}^{T}\left(t_{k}^{-}\right) y_{i}\left(t_{k}^{-}\right) .
\end{aligned}
$$

For any $k \in \mathbb{N}_{+}$, let $\varphi\left(t_{k}^{-}\right)=\min \left\{\left|y_{i}\left(t_{k}^{-}\right)\right|: i \in \mathscr{D}\left(t_{k}\right)\right\}$ and $\psi\left(t_{k}^{-}\right)=\max \left\{\left|y_{i}\left(t_{k}^{-}\right)\right|: i \notin \mathscr{D}\left(t_{k}\right)\right\}$. According to the selection of parameters in set $\mathscr{D}\left(t_{k}\right)$, we have $\varphi\left(t_{k}^{-}\right) \geq \psi\left(t_{k}^{-}\right)$. Since $\rho=1+l / n \cdot \mu(\mu+2) \in(0,1)$, we get $(1-\rho)(n-l)=$ $\left[\rho-(1+\mu)^{2}\right] l$. Hence, one has

$$
\begin{aligned}
(1-\rho) \sum_{i \notin \mathscr{D}\left(t_{k}\right)} y_{i}^{T}\left(t_{k}^{-}\right) y_{i}\left(t_{k}^{-}\right) \leq(1-\rho)(N-l)\left(\psi\left(t_{k}^{-}\right)\right)^{2} \\
\leq(1-\rho)(N-l)\left(\varphi\left(t_{k}^{-}\right)\right)^{2} \leq l\left[\rho-(1+\mu)^{2}\right]\left(\left(\varphi\left(t_{k}^{-}\right)\right)^{2}\right. \\
\quad \leq\left[\rho-(1+\mu)^{2}\right] \sum_{i \in \mathscr{D}\left(t_{k}\right)} y_{i}^{T}\left(t_{k}^{-}\right) y_{i}\left(t_{k}^{-}\right),
\end{aligned}
$$

which follows that

$$
\begin{aligned}
& (1+\mu)^{2} \sum_{i \in \mathscr{D}\left(t_{k}\right)} y_{i}^{T}\left(t_{k}^{-}\right) y_{i}\left(t_{k}^{-}\right)+\sum_{i \notin \mathscr{D}\left(t_{k}\right)} y_{i}^{T}\left(t_{k}^{-}\right) y_{i}\left(t_{k}^{-}\right) \\
& \quad \leq \rho \sum_{i=1}^{n} y_{i}^{T}\left(t_{k}^{-}\right) y_{i}\left(t_{k}^{-}\right) .
\end{aligned}
$$

From (41) to (43), we have 


$$
V\left(t_{k}^{+}\right) \leq \rho V\left(t_{k}^{-}\right)
$$

For any $\varepsilon>0$, let $\nu(t)$ be a unique solution of the following impulsive delayed system:

$$
\begin{cases}\dot{v}(t)=p \nu(t)+q \nu\left(t-\tau_{m}(t)\right)+\varepsilon, & t \neq t_{k}, \\ v\left(t_{k}^{+}\right)=\rho v\left(t_{k}^{-}\right), & t=t_{k} k \in \mathbb{N}_{+}, \\ \nu(t)=|\phi(t)|^{2}, & -\tau \leq t \leq 0 .\end{cases}
$$

According to Lemma 2, we can get

$$
\nu(t) \geq V(t), \quad t \geq 0 .
$$

By the formula for the variation of parameters, it follows from (45) that

$$
\nu(t)=w(t, 0) v(0)+\int_{0}^{t} w(t, s)[q \nu(s-\tau(s)+\varepsilon] \mathrm{d} s,
$$

where $w(t, s), \quad t>s \geq 0$, is the Cauchy matrix of the linear system

$$
\begin{cases}\dot{z}(t)=p z(t), & t \neq t_{k}, \\ z\left(t_{k}^{+}\right)=\rho z\left(t_{k}^{-}\right), & t=t_{k} k \in \mathbb{N}_{+} .\end{cases}
$$

According to the representation of the Cauchy matrix, since $0<\rho<1$ and $t-s / T_{a}-N_{0} \leq N_{\zeta}(t, s) \leq t-s / T_{a}+N_{0}$, we can obtain the following estimation:

$$
\begin{aligned}
w(t, s) & =e^{p(t-s)} \prod_{s<t_{k}<t} \rho \leq e^{p(t-s)} \rho^{N_{\zeta}(t, s)} \leq e^{p(t-s)} \rho^{t-s / T_{a}-N_{0}} \\
& =M_{1} e^{\eta_{2}(t-s)}
\end{aligned}
$$

where $M_{1}=\rho^{-N_{0}}$ and $\eta_{2}=p+\ln \rho / T_{a}$. Let $M_{2}=M_{1}$ $\sup _{-\tau \leq s \leq 0}|\phi(s)|^{2}$. Then, it can be derived from (45) and (49) that

$$
\nu(t) \leq M_{2} e^{\eta_{2} t}+\int_{0}^{t} M_{1} e^{\eta_{2}(t-s)}[q \nu(s-\tau(s))+\varepsilon] \mathrm{d} s .
$$

Define $h(v)=v+\eta_{2}+M_{1} q e^{v \tau}$. It follows from (38) that $h(0)<0$. Since $h(+\infty)=+\infty$ and $\dot{h}(v)>0$, there exists unique $\lambda>0$ such that

$$
\lambda+\eta_{2}+M_{1} q e^{\lambda \tau}=0
$$

On the contrary, it is obvious from (38) that $M_{1}^{-1} \eta_{2}+q<0$. Hence,

$$
\nu(t)=|\phi(t)|^{2}<M_{2} e^{-\lambda t}-\frac{\varepsilon M_{1}}{\eta_{2}+M_{1} q}, \quad-\tau \leq t \leq 0 .
$$

Then, we claim

$$
\nu(t)<M_{2} e^{-\lambda t}-\frac{\varepsilon M_{1}}{\eta_{2}+M_{1} q} .
$$

If inequality (51) is not true, there exists $t^{*}>0$ such that

$$
\begin{gathered}
\nu\left(t^{*}\right) \geq M_{2} e^{-\lambda t^{*}}-\frac{\varepsilon M_{1}}{\eta_{2}+M_{1} q}, \\
v(t)<M_{2} e^{-\lambda t}-\frac{\varepsilon M_{1}}{\eta_{2}+M_{1} q}, \quad t<t^{*} .
\end{gathered}
$$

From (50) and (55), we have

$$
\begin{aligned}
v\left(t^{*}\right) \leq & M_{2} e^{\eta_{2} t^{*}}+\int_{0}^{t^{*}} M_{1} e^{\eta_{2}\left(t^{*}-s\right)}[q \nu(s-\tau(s))+\varepsilon] \mathrm{d} s \\
< & e^{\eta_{2} t^{*}}\left\{M_{2}-\frac{\varepsilon M_{1}}{\eta_{2}+M_{1} q}+\int_{0}^{t^{*}} M_{1} e^{-\eta_{2} s}\right. \\
\cdot & {\left.\left[q\left(M_{2} e^{-\lambda(s-\tau(s))}-\frac{\varepsilon M_{1}}{\eta_{2}+M_{1} q}\right)+\varepsilon\right] \mathrm{d} s\right\} . }
\end{aligned}
$$

It is derived from (51) and (56) that

$$
\begin{aligned}
\nu\left(t^{*}\right)<e^{\eta_{2} t^{*}} & \left\{M_{2}-\frac{\varepsilon M_{1}}{\eta_{2}+M_{1} q}+M_{2} e^{-\left(\eta_{2}+\lambda\right) t^{*}}-M_{2}\right. \\
& \left.+\frac{\varepsilon M_{1}}{\eta_{2}+M_{1} q}-\frac{\varepsilon M_{1}}{\eta_{2}+M_{1} q} e^{-\eta_{2} t^{*}}\right\}=M_{2} e^{-\lambda t^{*}} \\
& -\frac{\varepsilon M_{1}}{\eta_{2}+M_{1} q},
\end{aligned}
$$

which contradicts with (54), and so, (53) holds. Letting $\varepsilon \longrightarrow 0$, one gets from (17) that

$$
V(t) \leq v(t) \leq M_{2} e^{-\lambda t}
$$

The proof is completed.

\section{Numerical Examples}

Two numerical examples are presented to demonstrate the validity of the above results in this section.

Example 1. Consider neural networks (5) with the following parameters:

$$
\begin{aligned}
C & =\left[\begin{array}{cc}
-3 & 0 \\
0 & -8
\end{array}\right], \\
B & =\left[\begin{array}{ll}
0.3 & 0.1 \\
0.2 & 0.2
\end{array}\right], \\
D & =\left[\begin{array}{ll}
0.4 & 0.2 \\
0 & 0.2
\end{array}\right], \\
L & =\left[\begin{array}{ll}
0.1 & 0 \\
0 & 0.2
\end{array}\right],
\end{aligned}
$$

and $f(y(t))=\tanh (0.8 y(t)), \tau(t)=e^{t} / 1+e^{t}$, where $y(t)=$ $\left(y_{1}(t), y_{2}(t)\right)^{T}$ is the state vector of the neural networks. By 
calculation, we can easily know $\eta_{1}+M_{0} q=-3.5126$. Figure 1 represents a hybrid impulsive sequence where $T_{a}=0.2, N_{0}=5, N_{1}=2$, and $\alpha=1.2$. The effect of the hybrid impulsive sequence is destabilizing.

According to Theorem 1, neural networks (5) with timevarying impulses will be globally exponentially stable if condition (10) is satisfied. Our numerical simulation draws the conclusion of Figure 2. From Figure 2, it can be observed that the state response of the neural networks tends to be stable quickly, which means the simulation results are consistent with the theory analysis.

Example 2. Consider neural networks (5) with

$$
\begin{aligned}
C & =\left[\begin{array}{cccccc}
1 & 0 & 0 & 0 & 0 & 0 \\
0 & -4 & 0 & 0 & 0 & 0 \\
0 & 0 & -1 & 0 & 0 & 0 \\
0 & 0 & 0 & -3 & 0 & 0 \\
0 & 0 & 0 & 0 & -2 & 0 \\
0 & 0 & 0 & 0 & 0 & -3
\end{array}\right], \\
B & =\left[\begin{array}{cccccc}
0.08 & 0 & -0.1 & 0 & 0 & 0 \\
0 & 0.02 & 0 & 0.1 & -0.3 & 0 \\
-0.05 & 0 & -0.1 & 0.5 & 0 & 0.4 \\
0.4 & 0.2 & -0.3 & 0.6 & -0.2 & 0.1 \\
0 & 0 & 0 & 0.2 & -0.3 & 0 \\
0.1 & -0.2 & 0 & 0 & 0.5 & -0.2
\end{array}\right],
\end{aligned}
$$$$
D=\left[\begin{array}{cccccc}
0.2 & 0.1 & 0 & 0 & 0 & 0 \\
0 & 0.1 & 0.2 & 0.3 & 0 & 0 \\
0.1 & 0 & -0.2 & 0 & 4 & 0.2 \\
0 & 0.1 & 0 & 0.1 & 0.2 & 0.2 \\
0 & 0.1 & 0.2 & 0.3 & 0 & 0 \\
0.2 & 0.1 & 0.3 & 0 & 0 & 0.2
\end{array}\right] \text {, }
$$$$
L=\left[\begin{array}{llllll}
1 & 0 & 0 & 0 & 0 & 0 \\
0 & 1 & 0 & 0 & 0 & 0 \\
0 & 0 & 1 & 0 & 0 & 0 \\
0 & 0 & 0 & 1 & 0 & 0 \\
0 & 0 & 0 & 0 & 1 & 0 \\
0 & 0 & 0 & 0 & 0 & 1
\end{array}\right],
$$

and $f(y(t))=\tanh (0.8 y(t)), \tau(t)=e^{t} / 1+e^{t}$, where $y(t)=$ $\left(y_{1}(t), y_{2}(t), \ldots, y_{6}(t)\right)^{T}$ is the state vector of the neural

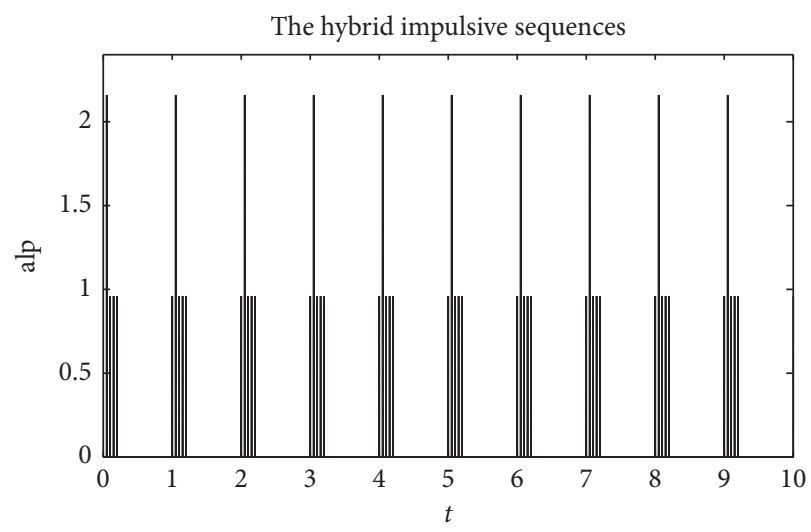

FIgURE 1: The hybrid impulsive sequence with $T_{a}=0.2, N_{0}=5, N_{1}=2$, and $\alpha=1.2$.

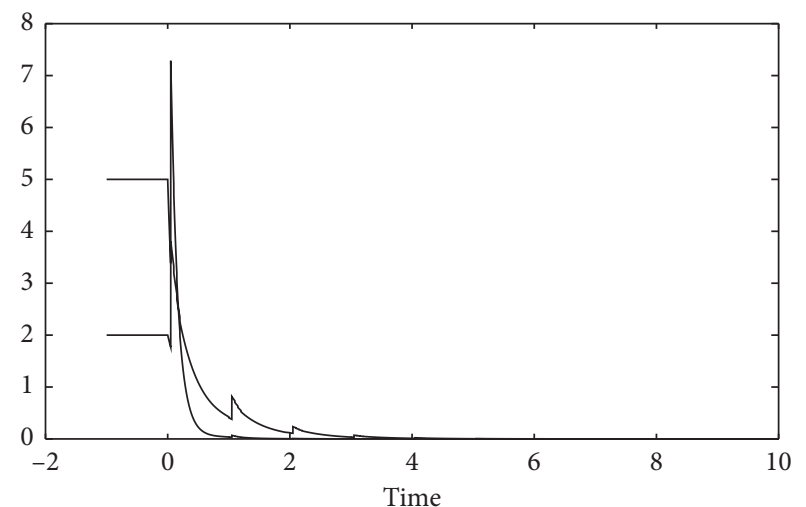

Figure 2: The state response of the neural networks in Theorem 1.

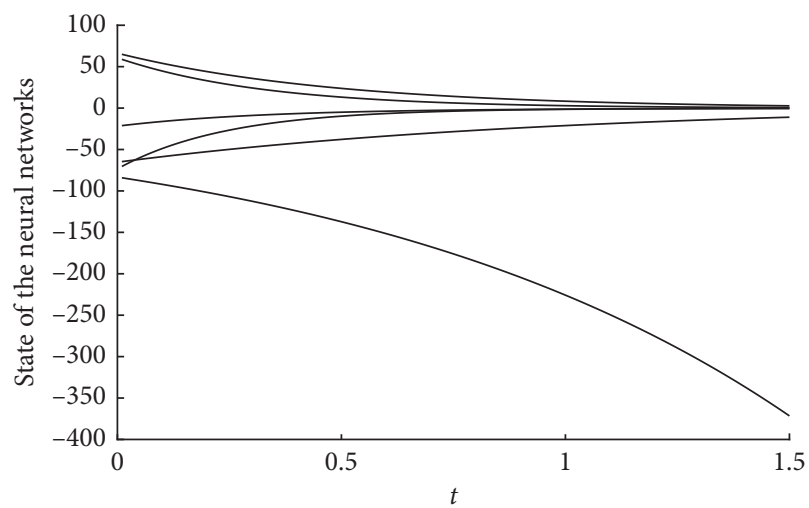

FIgURE 3: The state response of neural networks (5) without any controller in Theorem 2.

networks. The state response of neural networks (5) tends to be unstable without any controller, as is depicted in Figure 3. We select controller (35) with $T_{a}=0.25, N_{0}=$ $3, \mu=-1.5, l=3$, and $n=6$. By calculation, we can easily know $\eta_{2}+M_{1} q=-4.570469$. According to Theorem 2, the 


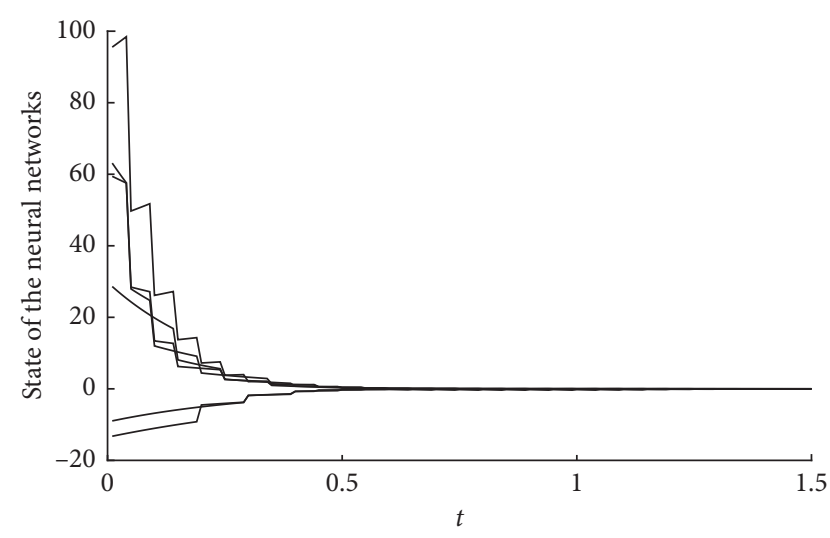

FIGURE 4: Simulation result for stabilization in Theorem 2.

pinning impulsive controller ensures global exponential stability of the considered neural networks. Simulation results approve the declared property (see Figure 4).

\section{Conclusion}

In this paper, the stability and stabilization problems of delayed neural networks with hybrid impulses have been studied. Based on Lyapunov stability theory combined with the comparison principle and the conception of average impulsive strength, a criterion for the exponential stability of delayed neural networks with hybrid impulses has been proposed. Then, a pinning impulsive controller has been designed to globally and exponentially stabilize the delayed neural networks with hybrid impulses. By revising the proof of the main results, some methods related to the concepts of average impulsive interval and average impulsive strength have been used to make the theoretical results less conservative. The derived stabilization criterion and the convergence rate are closely related with the proportion of the controlled neurons, time delay, impulsive strengths, and average impulsive interval of the neural networks. The validity of the theoretical results has been well explained by simulation results. In the future research, finite-time stabilization, persistent dwell-time, and state constraints will be included.

\section{Data Availability}

This paper is a theoretical paper and does not need data analysis. The data used to support the findings of this study are cited at relevant places within the article.

\section{Conflicts of Interest}

The authors declare that they have no conflicts of interest.

\section{Acknowledgments}

This work was supported by the Zhejiang Provincial Natural Science Foundation of China (Grant no. LR20F020002) and the Fundamental Research Funds for the Central
Universities of Henan Province (Grant nos. 20B110018 and 19B110012).

\section{References}

[1] C. Huang, J. Lu, G. Zhai, J. Cao, G. Lu, and M. Perc, "Stability and stabilization in probability of probabilistic Boolean networks," IEEE Transactions on Neural Networks and Learning Systems, p. 1. In press, 2020.

[2] C. Huang, X. Zhang, H.-K. Lam, and S.-H. Tsai, "Synchronization analysis for nonlinear complex networks with reaction-diffusion terms using fuzzy-model-based approach," IEEE Transactions on Fuzzy Systems, p. 1. In press, 2020.

[3] K. Shi, J. Wang, Y. Tang, and S. Zhong, "Reliable asynchronous sampled-data filtering of T-S fuzzy uncertain delayed neural networks with stochastic switched topologies," Fuzzy Sets and Systems, vol. 381, no. 15, pp. 1-25, 2020.

[4] C. Zhao, S. Zhong, X. Zhang, Q. Zhong, and K. Shi, "Novel results on nonfragile sampled-data exponential synchronization for delayed complex dynamical networks," International Journal of Robust and Nonlinear Control, vol. 30, no. 10, pp. 4022-4042, 2020.

[5] R. Olfati-Saber and R. M. Murray, "Consensus problems in networks of agents with switching topology and time-delays," IEEE Transactions on Automatic Control, vol. 49, no. 9, pp. 1520-1533, 2004.

[6] S. H. Strogatz, "Exploring complex networks," Nature, vol. 410, no. 6825 , pp. 268-276, 2001.

[7] M. E. J. Newman, "The structure and function of complex networks," SIAM Review, vol. 45, no. 2, pp. 167-256, 2003.

[8] Y. Y. Wang, W. X. Zhou, J. Luo, H. C. Yan, H. Y. Pu, and Y. Peng, "Reliable intelligent path following control for a robotic airship against sensor faults," IEEE/ASME Transactions on Mechatronics, vol. 24, no. 6, pp. 2572-2582, 2020.

[9] A. Chandrasekar, R. Rakkiyappan, J. Cao, and S. Lakshmanan, "Synchronization of memristor-based recurrent neural networks with two delay components based on second-order reciprocally convex approach," Neural Networks, vol. 57, pp. 79-93, 2014.

[10] Y. Xia and J. Wang, "A general projection neural network for solving monotone variational inequalities and related optimization problems," IEEE Transactions on Neural Networks, vol. 15, no. 2, pp. 318-328, 2004.

[11] Y. S. Xia and J. Wang, "A dual neural network for kinematic control of redundant robot manipulators," IEEE Transactions on Systems Man \& Cybernetics Part B Cybernetics A Publication of the IEEE Systems Man \& Cybernetics Society, vol. 31, no. 1, pp. 147-154, 2001.

[12] S. Y. Zhu, J. G. Lou, Y. Liu, Y. Y. Li, and Z. Wang, "Eventtriggered Control for the Stabilization of Probabilistic Boolean Control Networks," Complexity, vol. 2018, Article ID 9259348, 7 pages, 2018.

[13] X. Yang, B. Chen, Y. Li, Y. Liu, and F. E. Alsaadi, “Stabilization of dynamic-algebraic Boolean control networks via state feedback control," Journal of the Franklin Institute, vol. 355, no. 13, pp. 5520-5533, 2018.

[14] Y. Li, B. Li, Y. Liu, J. Lu, Z. Wang, and F. E. Alsaadi, "Set stability and stabilization of switched boolean networks with state-based switching," IEEE Access, vol. 6, pp. 35624-35630, 2018.

[15] Y. Li, J. Lou, Z. Wang, and F. E. Alsaadi, "Synchronization of dynamical networks with nonlinearly coupling function under hybrid pinning impulsive controllers," Journal of the Franklin Institute, vol. 355, no. 14, pp. 6520-6530, 2018. 
[16] Z.-H. Guan and G. Chen, "On delayed impulsive Hopfield neural networks," Neural Networks, vol. 12, no. 2, pp. 273-280, 1999.

[17] J. Lu, D. W. C. Ho, J. Cao, and J. Kurths, "Exponential synchronization of linearly coupled neural networks with impulsive disturbances," IEEE Transactions on Neural Networks, vol. 22, no. 2, pp. 329-336, 2011.

[18] Y. Q. Wang, J. Q. Lu, and Y. J. Lou, "Halanay-type inequality with delayed impulses and its applications," Science China, vol. 62, no. 9, pp. 192-206, 2019.

[19] J. Hu, G. Sui, X. Lv, and X. Li, "Fixed-time control of delayed neural networks with impulsive perturbations," Nonlinear Analysis: Modelling and Control, vol. 23, no. 6, pp. 904-920, 2018.

[20] Z. G. Zeng, J. Wang, and X. X. Liao, "Global exponential stability of a general class of recurrent neural networks with time-varying delays," IEEE Transactions on Circuits \& Systems I Fundamental Theory \& Applications, vol. 50, no. 10, pp. 1353-1358, 2003.

[21] Z. Zeng and J. Wang, "Improved conditions for global exponential stability of recurrent neural networks with timevarying delays," IEEE Transactions on Neural Networks, vol. 17 , no. 3, pp. 623-635, 2006.

[22] J. Cao and D. W. C. Ho, "A general framework for global asymptotic stability analysis of delayed neural networks based on LMI approach," Chaos, Solitons \& Fractals, vol. 24, no. 5, pp. 1317-1329, 2005.

[23] S. Arik, "An analysis of exponential stability of delayed neural networks with time varying delays," Neural Networks, vol. 17, no. 7, pp. 1027-1031, 2004.

[24] X. Li and J. Cao, "Adaptive synchronization for delayed neural networks with stochastic perturbation," Journal of the Franklin Institute, vol. 345, no. 7, pp. 779-791, 2008.

[25] Y. Zhang and Q.-L. Han, "Network-based synchronization of delayed neural networks," IEEE Transactions on Circuits and Systems I: Regular Papers, vol. 60, no. 3, pp. 676-689, 2013.

[26] Z. Wang, D. W. C. Ho, and X. Liu, "State estimation for delayed neural networks," IEEE Transactions on Neural Networks, vol. 16, no. 1, pp. 279-284, 2005.

[27] K. Shi, Y. Tang, S. Zhong, C. Yin, X. Huang, and W. Wang, "Nonfragile asynchronous control for uncertain chaotic Lurie network systems with Bernoulli stochastic process," International Journal of Robust and Nonlinear Control, vol. 28, no. 5, pp. 1693-1714, 2018.

[28] C. Li, Y. Y. Shen, and G. Feng, "Stabilizing effects of impulses in delayed BAM neural networks," IEEE Transactions on Circuits and Systems II: Express Briefs, vol. 55, no. 12, pp. 1284-1288, 2008.

[29] C. Li, C. Li, and C. Liu, "Destabilizing effects of impulse in delayed BAM neural networks," Modern Physics Letters B, vol. 23, no. 29, pp. 3503-3513, 2009.

[30] C. Li, S. Wu, G. G. Feng, and X. Liao, "Stabilizing effects of impulses in discrete-time delayed neural networks," IEEE Transactions on Neural Networks, vol. 22, no. 2, pp. 323-329, 2011.

[31] L. Pan and J. Cao, "Robust stability for uncertain stochastic neural network with delay and impulses," Neurocomputing, vol. 94, no. 3, pp. 102-110, 2012.

[32] T. Yang, L.-B. Yang, and C.-M. Yang, "Impulsive control of Lorenz system," Physica D: Nonlinear Phenomena, vol. 110, no. 1-2, pp. 18-24, 1997.

[33] J. Cao, D. W. C. Ho, and Y. Yang, "Projective synchronization of a class of delayed chaotic systems via impulsive control," Physics Letters A, vol. 373, no. 35, pp. 3128-3133, 2009.
[34] S. Chen, Q. Yang, and C. Wang, "Impulsive control and synchronization of unified chaotic system," Chaos, Solitons \& Fractals, vol. 20, no. 4, pp. 751-758, 2004.

[35] Z. Yang and D. Xu, "Stability analysis and design of impulsive control systems with time delay," IEEE Transactions on Automatic Control, vol. 52, no. 8, pp. 1448-1454, 2007.

[36] K. Udhayakumar, R. Rakkiyappan, J.-d. Cao, and X.-g. Tan, "Mittag-Leffler stability analysis of multiple equilibrium points in impulsive fractional-order quaternion-valued neural networks," Frontiers of Information Technology \& Electronic Engineering, vol. 21, no. 2, pp. 234-246, 2020.

[37] X. Li, T. Caraballo, R. Rakkiyappan, and X. Han, "On the stability of impulsive functional differential equations with infinite delays," Mathematical Methods in the Applied Sciences, vol. 38, no. 14, pp. 3130-3140, 2015.

[38] X. Li, J. Shen, and R. Rakkiyappan, "Persistent impulsive effects on stability of functional differential equations with finite or infinite delay," Applied Mathematics and Computation, vol. 329, pp. 14-22, 2018.

[39] X. Yang, X. D. Li, X. Xi, and P. Duan, "Review of stability and stabilization for impulsive delayed systems," Mathematical Biosciences \& Engineering, vol. 15, no. 6, pp. 1495-1515, 2018.

[40] X. Li, X. Yang, and T. Huang, "Persistence of delayed cooperative models: impulsive control method," Applied Mathematics and Computation, vol. 342, pp. 130-146, 2019.

[41] X. Li, D. O'Regan, and H. Akca, "Global exponential stabilization of impulsive neural networks with unbounded continuously distributed delays," IMA Journal of Applied Mathematics, vol. 80, no. 1, pp. 85-99, 2015.

[42] J. Lu, D. W. C. Ho, and J. Cao, "A unified synchronization criterion for impulsive dynamical networks," Automatica, vol. 46, no. 7, pp. 1215-1221, 2010.

[43] R. Rakkiyappan, D. Gayathri, G. Velmurugan, and J. Cao, "Exponential synchronization of inertial memristor-based neural networks with time delay using average impulsive interval approach," Neural Processing Letters, vol. 50, no. 3, pp. 2053-2071, 2019.

[44] J. Lu, J. Kurths, J. Cao, N. Mahdavi, and C. Huang, "Synchronization control for nonlinear stochastic dynamical networks: pinning impulsive strategy," IEEE Transactions on Neural Networks and Learning Systems, vol. 23, no. 2, pp. 285-292, 2012.

[45] P. Liu, L. Li, K. Shi, and J. Lu, "Pinning stabilization of probabilistic boolean networks with time delays," IEEE Access, vol. 8, pp. 154050-154059, 2020.

[46] Y. Li, "Impulsive synchronization of stochastic neural networks via controlling partial states," Neural Processing Letters, vol. 46, no. 1, pp. 59-69, 2017.

[47] X. Ji, J. Lu, J. Lou, J. Qiu, and K. Shi, “A unified criterion for global exponential stability of quaternion-valued neural networks with hybrid impulses," International Journal of Robust and Nonlinear Control, vol. 30, pp. 8098-8116, 2020, In press.

[48] N. Wang, X. Li, J. Lu, and F. E. Alsaadi, "Unified synchronization criteria in an array of coupled neural networks with hybrid impulses," Neural Networks, vol. 101, pp. 25-32, 2018.

[49] Y. Wang, H. Shen, and D. Duan, "On stabilization of quantized sampled-data neural-network-based control systems," IEEE Transactions on Cybernetics, vol. 47, no. 10, pp. 3124-3135, 2017. 